\title{
Immunohistochemical analysis of cell death pathways in gastrointestinal adenocarcinoma
}

\author{
Michiko Shintani ${ }^{1}$, Akiko Sangawa ${ }^{2}$, Naoki Yamao $^{3}$, Toshihiko Miyake ${ }^{4}$, and Shingo Kamoshida ${ }^{1}$ \\ ${ }^{1}$ Laboratory of Pathology, Division of Medical Biophysics, Kobe University Graduate School of Health Sciences, Kobe, Japan; ${ }^{2}$ De- \\ partment of Diagnostic Pathology, Osaka Red Cross Hospital, Osaka, Japan; ${ }^{3}$ Department of Clinical Laboratory; and ${ }^{4}$ Department of \\ Diagnostic Pathology, Kobe Central Hospital of Social Insurance, Kobe, Japan
}

(Received 6 October 2011; and accepted 14 October 2011)

\begin{abstract}
Caspase-8 and caspase-9 play crucial roles in the extrinsic and intrinsic apoptotic pathways, respectively. The nuclear translocation of apoptosis-inducing factor (AIF) is involved in caspase-independent apoptosis. Microtubule-associated protein 1 light chain 3 (LC3) plays a pivotal role in autophagy. In the present study, we analyzed the expression of cleaved caspase-8 (CC8), cleaved caspase-9 (CC9), AIF, and LC3 in 160 gastrointestinal adenocarcinomas. The nuclear expression of AIF was rare. The expression of CC8 in gastric and colorectal adenocarcinomas did not differ, whereas the percentage of $\mathrm{CC} 9$-positive tumors in gastric adenocarcinomas was significantly higher than in colorectal adenocarcinomas. In contrast, the percentage of LC3-positive tumors in gastric adenocarcinomas was significantly lower than in colorectal adenocarcinomas. CC8 and CC9 occasionally co-existed in the same tumor cells in gastric adenocarcinoma. However, LC3-positive tumor cells in colorectal adenocarcinomas were constantly negative for CC8. No correlation was identified between the expression of any markers and clinicopathological parameters. These results suggest that different cell death pathways are activated in a manner that depends upon the primary site and cell type. The extrinsic and intrinsic apoptotic pathways may be mutually regulated in gastric adenocarcinomas. Also, autophagy may function as a cellular guardian to avoid apoptosis in colorectal adenocarcinomas.
\end{abstract}

Apoptosis is a fundamental biochemical cell death pathway characterized by several morphologic nuclear changes such as chromatin condensation and extensive fragmentation of chromosomal DNA (7).

Aberrant apoptosis is an important component of many pathologic conditions, such as neoplastic, neurodegenerative, and autoimmune diseases $(4,20)$. Apoptosis is executed via a proteolytic cascade, which is catalyzed by the caspase family of cysteine

Address correspondence to: Michiko Shintani, PhD, Laboratory of Pathology, Division of Medical Biophysics, Kobe University Graduate School of Health Sciences, 7-10-2 Tomogaoka, Suma-ku, Kobe, Hyogo 654-0142, Japan

Tel: +81-78-796-4581, Fax: +81-78-796-4547

E-mail: mtshin@kobe-u.ac.jp proteases $(10,24)$. Caspases are synthesized as their proforms (procaspases) and are activated by selective proteolysis at an aspartate cleavage site. Two initiator pathways are involved in the activation of caspase-dependent apoptosis $(10,24)$. The extrinsic pathway is induced through the activation of death receptors by their respective ligands. This activation leads to the recruitment of adaptor proteins and activation of procaspase-8, i.e., formation of cleaved (active) caspase-8 (CC8). The intrinsic pathway is triggered by the release of cytochrome $c$ from mitochondria. Cytochrome $c$ interacts with apoptotic protease-activating factor 1 along with other cofactors and binds procaspase-9, thereby forming an apoptosome and releasing cleaved (active) caspase- 9 (CC9). CC8 and CC9 directly cleave and activate the downstream effector caspases including caspase-3. 
Cleavage of various intracellular proteins in apoptotic cells finally leads to disruption of cytoskeletal integrity, cell shrinkage, nuclear condensation, and the activation of endonucleases. The immunohistochemical expression of combination of caspases has been examined in gastrointestinal, pancreatic, gallbladder, non-small cell lung and breast carcinoma tissues, whereas each antibody used in the investigations should detect both the precursor and cleaved forms of the corresponding caspases $(9,19,23,25-29)$.

Apoptosis is not limited to the caspase-dependent pathways. Apoptosis-inducing factor (AIF), a mitochondrial protein, is involved in caspase-independent apoptosis. AIF is released from mitochondria and translocated first to the cytosol and then to the nucleus, where it participates in the processes of chromatin condensation and DNA fragmentation $(1,14)$

Autophagy plays an important role as a catabolic degradation system to protect cells against nutrient starvation and metabolic stresses $(2,17)$. During the autophagic process, the cytoplasmic components are sequestered and engulfed by double-membranebound structures known as autophagosomes. Autophagosomes are fused with lysosomes to form autolysosomes, in which the sequestered components are digested by lysosomal hydrolases. Microtubule-associated protein 1 light chain 3 (LC3) is an autophagosomal ortholog of yeast Atg8 $(2,17)$. The introduction of autophagy stimulates the upregulation of LC3 expression, and a cytosolic form of LC3 (LC3-I) is conjugated to phosphatidylethanolamine to form LC3-II, which is recruited to autophagosomal membranes. Subsequently, LC3-II is degraded by lysosomal hydrolases after the fusion of autophagosomes with lysosomes. Therefore, LC3 is a specific marker of autophagosome formation.

The immunohistochemical expression and distribution of various cleaved caspases and their relationships with caspase-independent apoptosis and autophagy have not previously been studied. In the present study, we analyzed cell death pathways in gastrointestinal adenocarcinomas by determining the expression of CC8, CC9, AIF, and LC3 in the tumor tissues using the specific antibodies. Further, to investigate the association of cell death pathways on tumor aggressiveness, we examined the correlation between the expression of each marker and clinicopathological parameters.

\section{MATERIALS AND METHODS}

Tissue samples. Eighty advanced gastric adenocarcinomas (42 well- to moderately differentiated and 38 poorly differentiated) and 80 advanced colorectal adenocarcinomas (70 well- to moderately differentiated and 10 poorly differentiated) were collected from the archives of the Department of Diagnostic Pathology of the Osaka Red Cross Hospital and the Kobe Central Hospital of Social Insurance. The study was approved by the local ethics committee. The tumors were classified according to the TNM classification of malignant tumors (22). Surgically resected tumor tissues were fixed in $10 \%$ formalin and embedded in paraffin. One or two paraffin blocks selected for immunohistochemistry contained tumor-non-tumor junctions, invasive edge, and viable tumors. Three-micrometer-thick sections were cut consecutively, and mounted on aminopropyltriethoxysilane-coated slides.

Antibodies. A rabbit monoclonal antibody against CC8 (cleaved at Asp391; clone 18C8) and a rabbit polyclonal antibody against AIF were purchased from Cell Signaling Technology (Danvers, MA, USA). A rabbit polyclonal antibody against LC3 was obtained from Medical \& Biological Laboratories (Nagoya, Japan).

A rabbit polyclonal antibody specific for CC9 (cleaved at Asp 330) was prepared as follows. An immunogen was produced by binding of the peptide of caspase-9 (RTFDQLD, 324-330) to bovine thyroglobulin. Two rabbits were immunized with $100 \mu \mathrm{g}$ of the immunogen followed by 7 subsequent injections with the same dose every 2 weeks. After confirming that the antiserum titer had risen sufficiently, whole blood was collected, and the antiserum was obtained by centrifugation. The antiserum was then purified by affinity chromatography on an immunogen peptide-solid phased column. Antibodies recognizing the pro-caspase-9 epitope in the antiserum were absorbed by addition of the peptide (RTFDQLDAISS, 324-334). The antiserum was tested for the presence of specific antibody against the peptide by an enzyme-linked immunosorbent assay.

Immunohistochemical staining. The sections were deparaffinized with xylene and rehydrated with a graduated series of ethanol solutions. Endogenous peroxidase was blocked by incubating the sections in $0.3 \%$ hydrogen peroxide in methanol for $30 \mathrm{~min}$. Heat-induced epitope retrieval was applied using a pressure cooker (T-FAL; Rumily, France) for $10 \mathrm{~min}$. Optimal soaking solutions, determined by preliminary experiments, were selected as follows: $1 \mathrm{mM}$ ethylenediaminetetraacetic acid solution, $\mathrm{pH} 8.0$ for CC8 and AIF, and $10 \mathrm{mM}$ citrate buffer, $\mathrm{pH} 6.0$ for 
LC3 and pH 7.0 for CC9. After pressure cooking, the sections were left at room temperature to cool in the soaking solution for $30 \mathrm{~min}$. The sections were washed in running tap water, followed by $0.01 \mathrm{M}$ phosphate buffered saline (PBS; pH 7.2), and then incubated overnight at room temperature in the primary antibody which reacts specifically with $\mathrm{CC} 8$, CC9, AIF or LC3. The sections were then rinsed in PBS. To detect CC8, AIF and LC3, the sections were incubated with the Histofine Simple Stain MAX-PO (Nichirei, Tokyo, Japan) for $1 \mathrm{~h}$ at room temperature. For detecting CC9, the sections were immunostained using a biotin-free catalyzed signal amplification system (CSA II; Dako Japan, Kyoto, Japan) according to the manufacturer's protocols. The reaction products were visualized with diaminobenzidine, and counterstained with Mayer's hematoxylin. Negative controls were performed by the omitting the primary antibodies. Sections known to be stained positively were included in each run as positive staining controls: lung adenocarcinoma for CC8, reactive lymphadenitis for CC9, breast adenocarcinoma for AIF, and cerebral infarction for LC3.

Immunostaining evaluation and statistical analysis. The immunostained sections were reviewed independently by three investigators (M.S., N.Y. and A.S.), who were blinded to patients' clinicopathological characteristics. All sections were observed by light microscopy with a high-power objective $(\times 40)$. In each field, 300-400 tumor cells were counted. The cytoplasmic or cytoplasmic/nuclear staining for CC8 and CC9, and the cytoplasmic staining for LC3 were considered positive. Based on the proportion of positively stained tumor cells in 10 selected representative fields of each section, CC8, CC9, and LC3 were regarded as positive if the staining was observed in $>15 \%,>5 \%$, and $>10 \%$ of tumor cells, respectively. These border values 15,5 , and 10 , were obtained from the trimmed mean value, which is best suited for data with uneven distribution. For evaluation of AIF immunoreactivity, the nuclear staining of the target cells was considered positive regardless of the intensity of cytoplasmic staining. Necrotic areas were excluded from the evaluation.

The chi-square test and Fisher's exact test were used to determine the statistical differences of marker expression between gastric adenocarcinomas and colorectal adenocarcinomas, or between well- to moderately differentiated and poorly differentiated adenocarcinomas, as well as the analysis of the correlations between marker expression and the age or gender of the patients, lymphatic invasion or vascular invasion. The correlations between marker expression and tumor location, depth of invasion, lymph node metastasis or pathological stage were analyzed using the Kruskal-Wallis test. Differences with $P$ values of $<0.05$ were considered significant.

\section{RESULTS}

Expression of cell death markers in neoplastic and non-neoplastic gastrointestinal tissues

The representative expression patterns of cell death markers in gastrointestinal adenocarcinomas are illustrated in Fig. 1. The immunostaining of CC8 and CC9 was either cytoplasmic or cytoplasmic/nuclear (Fig. 1a, b, d and e). The majority of tumor cells with nuclear staining of CC8 and CC9 show characteristics of apoptotic nuclei. The LC3 staining pattern was invariably granular cytoplasmic (Fig. 1i). Tumor cells with nuclear AIF staining were observed only rarely in all of the tumors examined, although cytoplasmic staining of AIF was usually shown. Few differences in the staining patterns of CC8, CC9, and LC3 were detected between invasive and non-invasive components within the same tumors.

Table 1 summarizes the immunohistochemical evaluation of CC8, CC9, and LC3 in 80 gastric adenocarcinomas and 80 colorectal adenocarcinomas. The immunopositivity of CC 8 was identified in 50\% of gastric and $54 \%$ of colorectal adenocarcinomas. However, this result is not statistically significant. There was no significant difference in the staining of well- to moderately and poorly differentiated gastric adenocarcinomas (50\% vs. 50\%), and well- to moderately differentiated and poorly differentiated colorectal adenocarcinomas (54\% vs. 50\%). While immunopositivity of CC9 was observed in $46 \%$ of gastric adenocarcinomas, it was only observed in $14 \%$ of colorectal adenocarcinomas $(P<0.0001)$. CC9 was positive in $45 \%$ of well- to moderately differentiated gastric adenocarcinoma and $47 \%$ of poorly differentiated gastric carcinomas, and in $10 \%$ of well- to moderately differentiated and $40 \%$ of poorly differentiated colorectal adenocarcinomas. These results were not found to be statistically significant. In contrast to CC9, $35 \%$ of colorectal adenocarcinomas were positive for LC3, while the staining of gastric adenocarcinomas was statistically less common $(6 \%, P<0.0001)$. LC3 immunopositivity was identified in $12 \%$ of well- to moderately differentiated gastric adenocarcinomas but not in poorly differentiated gastric carcinomas $(P<0.05)$, whereas there was no difference in well- to moder- 


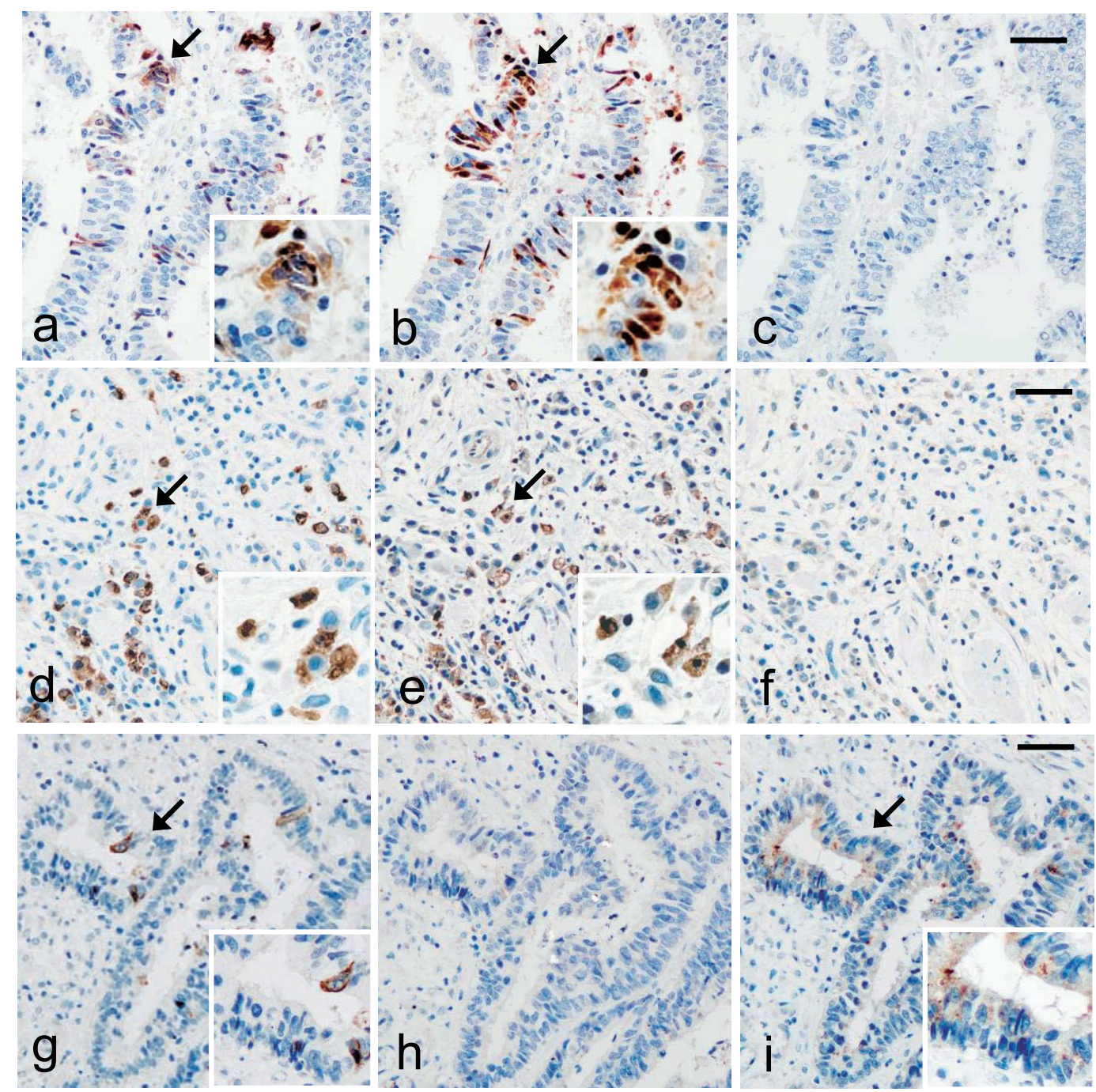

Fig. 1 Immunohistochemical staining for cleaved caspase-8 (CC8) (a, d, and g), cleaved caspase-9 (CC9) (b, e, and h), and microtubule-associated protein 1 light chain 3 (LC3) (c, f, and i) in the serial sections of gastrointestinal adenocarcinomas. In the case of well differentiated $(a-c)$ and poorly differentiated $(d-f)$ gastric adenocarcinomas, a number of the tumor cells are immunopositive for CC8 and CC9 in either the cytoplasm or the cytoplasm/nuclei, but no expression of LC3 is found. CC8 and CC9 co-exist occasionally in the tumor cells (arrows and insets). The case of well differentiated colonic adenocarcinoma ( $\mathrm{g}-\mathrm{i})$ shows immunopositivity for CC8 and LC3 but not for CC9. LC3-positive tumor cells reveal negative CC8 staining (arrows and insets). Calibration bar $=50 \mu \mathrm{m}$

ately differentiated and poorly differentiated colorectal adenocarcinomas ( $37 \%$ vs. $20 \%)$.

Epithelial cells on the surface of normal gastrointestinal mucosa and intestinal metaplastic mucosa were positive for CC8 and CC9. CC9 was also expressed in some of the lymphocytes and macrophages. Strong granular staining for LC3 was rarely demonstrated in the crypt epithelial cells of the normal colorectal and intestinal metaplastic mucosa. The expression of LC3 was also detected in the fundic glands of the stomach, as well as in mast cells and plasma cells. No nuclear AIF staining was found in any non-neoplastic cells.

Co-expression of plural cell death markers in gastrointestinal adenocarcinomas

Table 2 shows co-expression data for plural cell death markers within the same tumor tissues. CC8/ CC9 co-expression was found in $26 \%$ of gastric adenocarcinoma tissues and in $13 \%$ of colorectal adenocarcinoma tissues $(P<0.05)$. The corresponding percentages for CC8/LC3 co-expression were $0 \%$ and $15 \%(P<0.001)$, and for CC9/LC3 co-expression were $4 \%$ and $3 \%$ (not statistically significant). 
Table 1 Expression of CC8, CC9, and LC3 in gastrointestinal adenocarcinomas

\begin{tabular}{lrrr}
\hline & CC8 & CC9 & LC3 \\
\hline Gastric adenocarcinoma $(\mathrm{n}=80)$ & $40(50 \%)$ & $37(46 \%)]^{\mathrm{a}}$ & $5(6 \%)$ \\
Well to moderately differentiated $(\mathrm{n}=42)$ & $21(50 \%)$ & $19(45 \%)$ & $5(12 \%)$ \\
Poorly differentiated $(\mathrm{n}=38)$ & $19(50 \%)$ & $18(47 \%)$ & $0(0 \%)$ \\
Colorectal adenocarcinoma $(\mathrm{n}=80)$ & $43(54 \%)$ & $11(14 \%)$ & $28(35 \%)$ \\
Well to moderately differentiated $(\mathrm{n}=70)$ & $38(54 \%)$ & $7(10 \%)$ & $26(37 \%)$ \\
Poorly differentiated $(\mathrm{n}=10)$ & $5(50 \%)$ & $4(40 \%)$ & $2(20 \%)$ \\
\hline
\end{tabular}

${ }^{\mathrm{a}} \mathrm{P}<0.0001 ;{ }^{\mathrm{b}} \mathrm{P}<0.0001 ;{ }^{\mathrm{c}} \mathrm{P}<0.05$

Table 2 Co-expression of plural cell death markers within the same tumor tissues

\begin{tabular}{llrr}
\hline & \multicolumn{3}{c}{ Co-expression of } \\
\hline Gastric adenocarcinoma $(\mathrm{n}=80)$ & CC8/CC9 & CC8/LC3 & CC9/LC3 \\
Colorectal adenocarcinoma $(\mathrm{n}=80)$ & $21(26 \%)]^{\mathrm{a}}$ & $0(0 \%)$ \\
\hline
\end{tabular}

${ }^{\text {a }} P<0.05 ;{ }^{\text {b }} P<0.001$

The serial sections of representative tumor tissues clearly demonstrated whether cell death markers are co-expressed in the same tumor cells. CC8 and CC9 were found to co-exist occasionally in gastric adenocarcinoma cells (Fig. 1a, b, d and e). However, colorectal adenocarcinoma cells positive for LC3 invariably exhibited negative CC8 staining (Fig. 1g and i).

Correlation between expression of cell death markers and clinicopathological parameters in gastrointestinal adenocarcinomas

To investigate the association of cell death pathways on tumor aggressiveness, we examined the correlation between expression of CC8, CC9, and LC3 and clinicopathological parameters including age, gender, location, depth of invasion, lymph node metastasis, lymphatic invasion, vascular invasion, and pathological stage (Tables 3 and 4). However, in both gastric and colorectal adenocarcinomas, none of the clinicopathological parameters were associated with the expression of CC8, CC9, or LC3 nor the co-expression of CC8/CC9 or CC8/LC3.

\section{DISCUSSION}

The present study was undertaken to investigate the expression of the cell death markers CC8, CC9, AIF, and LC3 in gastrointestinal adenocarcinomas using specific antibodies, and to determine the correlation between marker expression and clinicopathological parameters. There was no difference in the expression of CC8 between gastric and colorectal adenocarcinomas, whereas the percentage of tumors positive for CC9 in gastric adenocarcinomas was significantly higher than in colorectal adenocarcinomas $(P<0.0001)$. It has been shown that the expression of caspase- 8 and caspase- 9 in gastrointestinal adenocarcinomas is observed with a much higher frequency $(23,29)$. Each antibody used in the previous studies, however, could not discriminate between the active and inactive forms of the caspases. Only the active form is responsible for the effects of the caspases leading to apoptosis. We also found that non-neoplastic epithelial cells in gastrointestinal mucosa were positive for CC8 and CC9, whereas some of the lymphocytes and macrophages were found to express CC9 but not CC8. These results strongly suggest that caspase- 8 and caspase- 9 are activated differentially in a manner that depends upon the primary site and cell type.

By applying the serial sectioning method, moreover, it was possible to determine that CC8 and CC9 co-exist occasionally in the same tumor cells in gastric adenocarcinomas. It is known that the BH3-domain protein Bid is cleaved by CC8 into truncated Bid, which induces cytochrome $c$ release leading to CC9 formation (15). These findings might suggest the existence of mutual regulation of the extrinsic and intrinsic apoptotic pathways in gastric adenocarcinomas. While an immunohistochemical study demonstrated heterogeneous expression of Bid between gastric adenocarcinomas (21), the expression of truncated Bid remains to be elucidated.

Caspase-independent cell death pathways include AIF-induced cell death and potentially autophagic cell death. Jeong et al. and Lee et al. reported AIF immunopositivity in $100 \%$ of colorectal and $70 \%$ of 
Table 3 Correlation between the expression of CC8, CC9, or LC3 and the clinicopathological parameters in gastric adenocarcinomas

\begin{tabular}{|c|c|c|c|c|}
\hline & CC8 & CC9 & LC3 & $\begin{array}{c}\text { CC8/CC9 } \\
\text { co-expression }\end{array}$ \\
\hline \multicolumn{5}{|l|}{ Age (years) } \\
\hline$<60(\mathrm{n}=11)$ & $5(45)$ & $5(45)$ & $1(9)$ & $3(27)$ \\
\hline$\geq 60(\mathrm{n}=69)$ & $35(51)$ & $32(46)$ & $4(6)$ & $18(26)$ \\
\hline \multicolumn{5}{|l|}{ Gender } \\
\hline Male $(n=57)$ & $29(51)$ & $26(46)$ & $5(9)$ & $13(23)$ \\
\hline Female $(\mathrm{n}=23)$ & $11(48)$ & $11(48)$ & $0(0)$ & $8(35)$ \\
\hline \multicolumn{5}{|l|}{ Location } \\
\hline Cardia (n = 19) & 7 (37) & 7 (37) & $2(11)$ & $3(16)$ \\
\hline Fundus $(\mathrm{n}=35)$ & $17(49)$ & $19(54)$ & $3(9)$ & $11(31)$ \\
\hline Antrum (n = 26) & $16(62)$ & $11(42)$ & $0(0)$ & $7(27)$ \\
\hline \multicolumn{5}{|l|}{ Depth of invasion* } \\
\hline pT2 (n = 17) & $11(65)$ & $8(47)$ & $1(6)$ & 5 (29) \\
\hline pT3 (n = 22) & $8(36)$ & $10(45)$ & $3(14)$ & $6(27)$ \\
\hline pT4 $(\mathrm{n}=41)$ & $21(51)$ & $19(46)$ & $1(2)$ & $10(24)$ \\
\hline \multicolumn{5}{|l|}{ Lymph node metastasis* } \\
\hline pN0 $(n=27)$ & $16(59)$ & $12(44)$ & $1(4)$ & $8(30)$ \\
\hline $\mathrm{pN} 1(\mathrm{n}=16)$ & $6(38)$ & $7(44)$ & $2(13)$ & $5(31)$ \\
\hline pN2 (n = 13) & $4(31)$ & $7(54)$ & $1(8)$ & $2(15)$ \\
\hline pN3 $(\mathrm{n}=24)$ & $14(58)$ & $11(46)$ & $1(4)$ & $6(25)$ \\
\hline \multicolumn{5}{|l|}{ Lymphatic invasion } \\
\hline Ly $(-)(\mathrm{n}=15)$ & $8(53)$ & $6(40)$ & $1(7)$ & $3(20)$ \\
\hline Ly $(+)(n=65)$ & 32 (49) & $31(48)$ & $4(6)$ & $18(28)$ \\
\hline \multicolumn{5}{|l|}{ Vascular invasion } \\
\hline $\mathrm{V}(-)(\mathrm{n}=41)$ & $24(59)$ & $20(49)$ & $1(2)$ & $12(29)$ \\
\hline $\mathrm{V}(+)(\mathrm{n}=39)$ & $16(41)$ & $17(44)$ & $4(10)$ & $9(23)$ \\
\hline \multicolumn{5}{|l|}{ UICC p-Stage* } \\
\hline I B $(\mathrm{n}=11)$ & $9(82)$ & $4(36)$ & $0(0)$ & $3(27)$ \\
\hline IIA and IIB $(n=26)$ & $11(42)$ & $12(46)$ & $4(15)$ & $8(31)$ \\
\hline IIIA, IIIB, and IIIC (n= 43) & $20(47)$ & $21(49)$ & $1(2)$ & $10(23)$ \\
\hline
\end{tabular}

* According to the TNM classification of malignant tumors, the numerals in parentheses indicate the percentage of positive tumors.

gastric adenocarcinomas, respectively $(6,11)$. However, only the cytoplasmic staining of AIF was evaluated in their studies, even though AIF execute caspase-independent apoptosis within the nucleus (1, 14). Our study revealed that nuclear AIF staining was rare, thereby suggesting the existence of a non-apoptotic role for AIF in gastrointestinal adenocarcinoma cells. According to the literature, the contribution of AIF to caspase-independent apoptosis is seen only when caspases are inhibited or inactivated (18).

In contrast to CC9, increased expression of LC3, a marker of autophagic activity, was seen in colorectal adenocarcinomas relative to gastric adenocarcinomas $(P<0.0001)$. The immunopositivity, however, was found to be rare in normal colorectal epithelium. The higher frequency of LC3 expression in colorectal adenocarcinomas compared with the corresponding normal tissue may indicate that the ex- pression of LC3 is associated with the development of colorectal carcinoma (30).

The present work also confirmed that LC3-positive tumor cells in colorectal adenocarcinomas were consistently negative for CC8. Caspase-dependent apoptosis is the predominant form of cell death induced by anticancer agents and tumor cells that can evade apoptosis should be resistant to chemotherapy (5). On the other hand, in addition to its role in cell death under high damage levels, autophagy promotes cell survival by degrading and recycling long-lived proteins and cellular components when the cell is exposed to limited conditions of stress (12). Autophagy has also been demonstrated recently as a protective mechanism against chemotherapy- or radiationinduced apoptosis. This process involves adapting cells to stress conditions and its inhibition could provide a promising strategy for cancer therapy (2). 
Table 4 Correlation between the expression of CC8, CC9, or LC3 and the clinicopathological parameters in colorectal adenocarcinomas

\begin{tabular}{|c|c|c|c|c|}
\hline & CC8 & CС9 & LC3 & $\begin{array}{c}\text { CC8/LC3 } \\
\text { co-expression }\end{array}$ \\
\hline \multicolumn{5}{|l|}{ Age (years) } \\
\hline$<60(\mathrm{n}=22)$ & $11(50)$ & $4(18)$ & $7(32)$ & $2(9)$ \\
\hline$\geq 60(\mathrm{n}=58)$ & $32(55)$ & $7(12)$ & $21(36)$ & $10(17)$ \\
\hline \multicolumn{5}{|l|}{ Gender } \\
\hline Male $(\mathrm{n}=45)$ & $24(53)$ & $5(11)$ & $19(42)$ & $6(13)$ \\
\hline Female $(\mathrm{n}=35)$ & $19(54)$ & $6(17)$ & $9(26)$ & $6(17)$ \\
\hline \multicolumn{5}{|l|}{ Location } \\
\hline Right colon $(n=24)$ & $16(67)$ & $2(8)$ & $8(33)$ & $6(25)$ \\
\hline Left colon $(n=26)$ & $11(42)$ & $4(15)$ & $11(42)$ & $2(8)$ \\
\hline Rectum $(\mathrm{n}=30)$ & $16(53)$ & $5(17)$ & $9(30)$ & $4(13)$ \\
\hline \multicolumn{5}{|l|}{ Depth of invasion* } \\
\hline pT2 (n = 8) & $3(38)$ & $1(13)$ & $2(25)$ & $1(13)$ \\
\hline pT3 (n = 47) & $27(57)$ & $5(11)$ & $19(40)$ & $7(15)$ \\
\hline pT4 $(\mathrm{n}=25)$ & $13(52)$ & $5(20)$ & 7 (28) & $4(16)$ \\
\hline \multicolumn{5}{|l|}{ Lymph node metastasis* } \\
\hline pN0 $(n=36)$ & $20(56)$ & $3(8)$ & $15(42)$ & 7 (19) \\
\hline $\mathrm{pN} 1(\mathrm{n}=32)$ & $17(53)$ & $7(22)$ & $11(34)$ & $5(16)$ \\
\hline pN2 (n = 12) & $6(50)$ & $1(8)$ & 2 (17) & $0(0)$ \\
\hline \multicolumn{5}{|l|}{ Lymphatic invasion } \\
\hline Ly $(-)(\mathrm{n}=25)$ & $13(52)$ & $3(12)$ & $11(44)$ & $6(24)$ \\
\hline Ly $(+)(n=55)$ & $30(55)$ & $8(15)$ & $17(31)$ & $6(11)$ \\
\hline \multicolumn{5}{|l|}{ Vascular invasion } \\
\hline $\mathrm{V}(-)(\mathrm{n}=40)$ & $21(53)$ & $5(13)$ & $17(43)$ & 9 (23) \\
\hline$V(+)(n=40)$ & $22(55)$ & $6(15)$ & $11(28)$ & $3(8)$ \\
\hline \multicolumn{5}{|l|}{ UICC p-Stage* } \\
\hline IB $(\mathrm{n}=7)$ & $3(43)$ & $1(14)$ & 2 (29) & $1(14)$ \\
\hline IIA and IIB $(\mathrm{n}=28)$ & $17(61)$ & $2(7)$ & $12(43)$ & $6(21)$ \\
\hline IIIA, IIIB, and IIIC $(\mathrm{n}=45)$ & $23(51)$ & $8(18)$ & $14(31)$ & $5(11)$ \\
\hline
\end{tabular}

* According to the TNM classification of malignant tumors, the numerals in parentheses indicate the percentage of positive tumors.

For example, the inhibition of autophagy enhances the therapeutic efficacy of 5-fluorouracil in colonic adenocarcinoma cells (13). It remains to be seen through specifically designed preclinical and clinical trials whether our findings will be useful in selecting autophagy inhibitor candidates when designing LC3 expression-based treatments.

The clinical implications of caspase-dependent or independent apoptosis and autophagy have not been fully studied. The present study indicated that there was no correlation between the expression of any marker in either gastric or colorectal adenocarcinomas and any of the clinicopathological parameters, including age, gender, differentiation, location, depth of tumor invasion, lymph node metastasis, lymphatic invasion, vascular invasion and pathological stage. This suggests that the activation of cell death pathways is not implicated in tumor aggressiveness. As described above, the activities of key proteins for each pathway may play an important role in cell death, which is associated with chemotherapy regimens $(3,5,8,13,16)$. Further investigation is therefore needed to clarify the contribution of CC8, CC9, AIF, and LC3 in gastrointestinal cancer prognosis or response to treatment.

In conclusion, we defined for the first time the expression patterns of CC8 and CC9 in gastrointestinal adenocarcinomas. In addition, immunopositivity for AIF and LC3 was evaluated simultaneously. The data obtained in the present study suggest that activation of different cell death pathways occurs in a manner that depends upon the primary site and cell type. The extrinsic and intrinsic apoptotic pathways may be mutually regulated in gastric adenocarcinomas. Also, autophagy may function as a cellular guardian to avoid caspase-dependent apoptosis in colorectal adenocarcinomas. 


\section{Acknowledgments}

We would like to thank Dr. Masayuki Shintaku for his kind support. This study was supported by a Grant-in-Aid for Scientific Research (No. 20590355) from the Japan Society for the Promotion of Science.

\section{REFERENCES}

1. Bröker LE, Kruyt FAE and Giaccone G (2005) Cell death independent of caspases: a review. Clin Cancer Res 11, 31553162.

2. Chen S, Rehman SK, Zhang W, Wen A, Yao L and Zhang J (2010) Autophagy is a therapeutic target in anticancer drug resistance. Biochim Biophys Acta 1806, 220-229.

3. Grivicich I, Regner A, da Rocha AB, Grass LB, Alves PA, Kayser GB, Schwartsmann G and Henriques JA (2005) Irinotecan/5-fluorouracil combination induces alterations in mitochondrial membrane potential and caspases on colon cancer cell lines. Oncol Res 15, 385-392.

4. Hanahan D and Weinberg RA (2000) The hallmarks of cancer. Cell 100, 57-70.

5. Houghton JA (1999) Apoptosis and drug response. Curr Opin Oncol 11, 475-481.

6. Jeong EG, Lee JW, Soung YH, Nam SW, Kim SH, Lee JY, Yoo NJ and Lee SH (2006) Immunohistochemical and mutational analysis of apoptosis-inducing factor (AIF) in colorectal carcinomas. APMIS 114, 867-873.

7. Kerr JFR, Winterford CM and Harmon BV (1994) Apoptosis. Its significance in cancer and cancer therapy. Cancer 73, 2013-2026.

8. Kim R, Tanabe K, Inoue H and Toge T (2002) Mechanism(s) of antitumor action in protracted infusion of low dose 5-fluorouracil and cisplatin in gastric carcinoma. Int $J$ Oncol 20, 549-555.

9. Koyama S (2003) Differential expression of intracellular apoptotic signaling molecules in tumor and tumor-infiltrating lymphocytes during development of invasion and/or metastasis of gastric carcinoma. Dig Dis Sci 48, 2290-2300.

10. Lavrik IN, Golks A and Krammer PH (2005) Caspases: pharmacological manipulation of cell death. J Clin Invest 115, 2665-2672.

11. Lee JW, Jeong EG, Soung YH, Kim SY, Nam SW, Kim SH, Lee JY, Yoo NJ and Lee SH (2006) Immunohistochemical analysis of apoptosis-inducing factor (AIF) expression in gastric carcinomas. Pathol Res Pract 202, 497-501.

12. Levine B and Klionsky DJ (2004) Development by selfdigestion: molecular mechanisms and biological functions of autophagy. Dev Cell 6, 463-477.

13. Li J, Hou N, Faried A, Tsutsumi S and Kuwano H (2010) Inhibition of autophagy augments 5-fluorouracil chemotherapy in human colon cancer in vitro and in vivo model. Eur $J$ Cancer 46, 1900-1909.

14. Lorenzo HK and Susin SA (2007) Therapeutic potential of AIF-mediated caspase-independent programmed cell death.
Drug Resist Updates 10, 235-255

15. Luo X, Budihardjo I, Zou H, Slaughter C and Wang X (1998) Bid, a Bcl2 interacting protein, mediates cytochrome c release from mitochondria in response to activation of cell surface death receptors. Cell 94, 481-490.

16. Marchetti P, Galla DA, Russo FP, Ricevuto E, Flati V, Porzio G, Ficorella C and Cifone MG (2004) Apoptosis induced by oxaliplatin in human colon cancer HCT15 cell line. Anticancer Res 24, 219-226.

17. Mathew R, Karantza-Wadsworth V and White E (2007) Role of autophagy in cancer. Nat Rev Cancer 7, 961-967.

18. Modjtahedi N, Giordanetto F, Madeo F and Kroemer G (2006) Apoptosis-inducing factor: vital and lethal. Trends Cell Biol 16, 264-272.

19. Palmerini F, Devilard E, Jarry A, Birg F and Xerri L (2001) Caspase 7 downregulation as an immunohistochemical marker of colonic carcinoma. Hum Pathol 32, 461-467.

20. Reed JC (2000) Mechanisms of apoptosis. Am J Pathol 157, 1415-1430.

21. Smith L, Berrieman HK, O’Kane SL, Campbell A, Maraveyas A and Cawkwell L (2006) Immunohistochemical detection of apoptotic markers in gastric cancer. Oncol Res 15, 441-444.

22. Sobin LH, Gospodarowicz MK and Wittekind C (eds) (2009) TNM Classification of Malignant Tumours. 7th edition. Wiley-Blackwell, New Jersey.

23. Sträter J, Herter I, Merkel G, Hinz U, Weitz J and Möller P (2010) Expression and prognostic significance of APAF-1, caspase-8 and caspase-9 in stage II/III colon carcinoma: caspase-8 and caspase-9 is associated with poor prognosis. Int $J$ Cancer 127, 873-880.

24. Thornberry NA and Lazebnik Y (1998) Caspases: enemies within. Science 281, 1312-1316.

25. Törmänen-Näpänkangas U, Soini Y, Kahlos K, Kinnula V and Pääkkö P (2001) Expression of caspases-3, -6 and -8 and their relation to apoptosis in non-small cell lung carcinoma. Int J Cancer 93, 192-198.

26. Turunen N, Pääkkö P and Soini Y (2000) Apoptosis in gallbladder carcinomas and dysplasias, its relation to the expression of caspases 3, 6 and 8 and apoptosis regulating proteins bcl-2, mcl-1 and bax. Histol Histopathol 15, 53-60.

27. Vakkala M, Pääkkö P and Soini Y (1999) Expression of caspases 3, 6 and 8 is increased in parallel with apoptosis and histological aggressiveness of the breast lesion. $\mathrm{Br} \mathrm{J}$ Cancer 81, 592-599.

28. Virkajärvi N, Pääkkö P and Soini Y (1998) Apoptotic index and apoptosis influencing proteins bcl-2, mcl-1, bax and caspases 3, 6 and 8 in pancreatic carcinoma. Histopathology 33, 432-439.

29. Yoo NJ, Kim HS, Kim SY, Park WS, Kim SH, Lee JY and Lee SH (2002) Stomach cancer highly expresses both initiator and effector caspases; an immunohistochemical study. APMIS 110, 825-832.

30. Yoshioka A, Miyata H, Doki Y, Yamasaki M, Sohma I, Gotoh K, Takiguchi S, Fujiwara Y, Uchiyama Y and Monden M (2008) LC3, an autophagosome marker, is highly expressed in gastrointestinal cancers. Int J Oncol 33, 461-468. 